\title{
Pattern-avoiding set partitions and Catalan numbers
}

\author{
Toufik Mansour \\ Department of Mathematics, University of Haifa, 31905 Haifa, Israel \\ tmansour@univ.haifa.ac.il \\ Mark Shattuck \\ Department of Mathematics, University of Haifa, 31905 Haifa, Israel \\ maarkons@excite.com
}

Submitted: Mar 29, 2011; Accepted: Feb 10, 2012; Published: Feb 23, 2012

Mathematics Subject Classification: 05A18, 05A15

Dedicated to Doron Zeilberger on the occasion of his sixtieth birthday

\begin{abstract}
We identify several subsets of the partitions of $[n]$, each characterized by the avoidance of a pair of patterns, respectively of lengths four and five. Each of the classes we consider is enumerated by the Catalan numbers. Furthermore, the members of each class having a prescribed number of blocks are enumerated by the Narayana numbers. We use both algebraic and combinatorial methods to establish our results. In some of the cases, we make use of the kernel method to solve the recurrence arising when a further statistic is considered. In other cases, we define bijections with previously enumerated classes which preserve the number of blocks. Two of our bijections are of an algorithmic nature and systematically replace the occurrences of one pattern with those of another having the same length.
\end{abstract}

\section{Introduction}

If $n \geq 1$, then a partition of $[n]=\{1,2, \ldots, n\}$ is any collection of non-empty, pairwise disjoint subsets, called blocks, whose union is $[n]$. (If $n=0$, then there is a single empty partition of $[0]=\varnothing$ which has no blocks.) A partition $\Pi$ having exactly $k$ blocks is called a $k$-partition. We will denote the set of all $k$-partitions of $[n]$ by $P_{n, k}$ and the set of all partitions of $[n]$ by $P_{n}$. A partition $\Pi$ is said to be in standard form if it is written as $\Pi=B_{1} / B_{2} / \cdots$, where the blocks are arranged in ascending order according to the size of the smallest elements. One may also represent $\Pi=B_{1} / B_{2} / \cdots \in P_{n}$, expressed in the standard form, equivalently as $\pi=\pi_{1} \pi_{2} \cdots \pi_{n}$, wherein $j \in B_{\pi_{j}}, 1 \leq j \leq n$, called the canonical sequential form; and, in such case, we will write $\Pi=\pi$. For example, the 
partition $\Pi=1,5,7 / 2,3 / 4,8 / 6 \in P_{8,4}$ has as its cano nical sequential form $\pi=12231413$. Note that $\pi=\pi_{1} \pi_{2} \cdots \pi_{n} \in P_{n, k}$ is a restricted growth function from $[n]$ to $[k]$ (see, e.g., [16] or [18] for details), meaning that it satisfies the following three properties: (i) $\pi_{1}=1$, (ii) $\pi$ is onto $[k]$, and (iii) $\pi_{i+1} \leq \max \left\{\pi_{1}, \pi_{2}, \ldots, \pi_{i}\right\}+1$ for all $i, 1 \leq i \leq n-1$. In what follows, we will represent set partitions as words using their canonical sequential forms and consider some particular cases of the general problem of counting the members of a partition class having various restrictions imposed on the order of the letters.

A classical pattern $\tau$ is a member of $[\ell]^{m}$ which contains all of the letters in $[\ell]$. We say that a word $\sigma \in[k]^{n}$ contains the classical pattern $\tau$ if $\sigma$ contains a subsequence that is order-isomorphic to $\tau$. Otherwise, we say that $\sigma$ avoids $\tau$. For example, a word $\sigma=\sigma_{1} \sigma_{2} \cdots \sigma_{n}$ avoids the pattern 231 if it has no subsequence $\sigma_{i} \sigma_{j} \sigma_{k}$ with $i<j<k$ and $\sigma_{k}<\sigma_{i}<\sigma_{j}$ and avoids the pattern 1221 if it has no subsequence $\sigma_{i} \sigma_{j} \sigma_{k} \sigma_{\ell}$ with $\sigma_{i}=\sigma_{\ell}<\sigma_{j}=\sigma_{k}$. The pattern avoidance question has been the topic of much research in enumerative combinatorics, starting with Knuth [6] and Simion and Schmidt [13] on permutations. See, for example, the papers by Noonan and Zeilberger [10], Robertson, Wilf and Zeilberger [11], and Mansour and Vainshtein [8]. More recently, the problem has been considered on further structures such as words, compositions, and finite set partitions. For the avoidance problem on set partitions, we refer the reader to the papers by Klazar [5], Sagan [12], and Jelínek and Mansour [4] and to the references therein.

We will use the following notation. Suppose $\left\{w_{1}, w_{2}, \ldots\right\}$ is a set of classical patterns. Let $P_{n}\left(w_{1}, w_{2}, \ldots\right)$ be the subset of $P_{n}$ consisting of those partitions whose sequential representations avoid $\left\{w_{1}, w_{2}, \ldots\right\}$, and let $P_{n, k}\left(w_{1}, w_{2}, \ldots\right)=P_{n, k} \cap P_{n}\left(w_{1}, w_{2}, \ldots\right)$. We will denote the cardinalities of $P_{n}\left(w_{1}, w_{2}, \ldots\right)$ and $P_{n, k}\left(w_{1}, w_{2}, \ldots\right)$ by $p_{n}\left(w_{1}, w_{2}, \ldots\right)$ and $p_{n, k}\left(w_{1}, w_{2}, \ldots\right)$, respectively. From the definitions, note that

$$
p_{n}\left(w_{1}, w_{2}, \ldots\right)=\sum_{k \geq 0} p_{n, k}\left(w_{1}, w_{2}, \ldots\right)
$$

The Catalan numbers $C_{n}$, named after the nineteenth century Belgian mathematician Eugene Catalan, have been shown to count many structures occurring in both enumerative and algebraic combinatorics. They seem to date to 1751, when Euler showed that the number of triangulations of an $n$-gon is given by the product $\frac{2 \cdot 6 \cdot 10 \cdots(4 n-10)}{2 \cdot 3 \cdots(n-1)}$, which is now called $C_{n-2}$. Perhaps the most fundamental structure counted by $C_{n}$ is the set of lattice paths from $(0,0)$ to $(2 n, 0)$ using up $(1,1)$ and down $(1,-1)$ steps that never dip below the $x$-axis (called Catalan or Dyck paths). Among the other important structures counted by $C_{n}$ are the plane binary trees having $n+1$ leaves, the standard Young tableaux of shape $(n, n-1)$, and the linear expansions of the poset $2 \times n$. In terms of avoidance, it is well known that $C_{n}$ counts the number of permutations of $[n]$ avoiding a single classical pattern $\tau$, where $\tau$ is any member of $S_{3}$ (see, e.g., [6] or [13]) as well as the number of partitions of $[n]$ avoiding either 1212 or 1221 (called non-crossing and nonnesting partitions, respectively; see, e.g., [5] or [4]). The $C_{n}$ have generating function (see (2.5.16) of [20])

$$
C(x)=\sum_{n \geq 0} C_{n} x^{n}=\frac{1-\sqrt{1-4 x}}{2 x}
$$


and are given by the closed form $C_{n}=\frac{1}{n+1}\left(\begin{array}{c}2 n \\ n\end{array}\right)$. To date, there are at least 190 structures known to be enumerated by the Catalan numbers; see Stanley's website [15] for a complete list.

The Narayana numbers $N_{n, k}$ count the set of Catalan paths of semilength $n$ having exactly $k$ peaks. Among their other combinatorial interpretations is that they count the number of 132-avoiding permutations of size $n$ having $k-1$ descents as well as the number of full binary trees with $n$ internal nodes and $k-1$ jumps. The numbers $N_{n, k}$ also count the non-crossing (or non-nesting) partitions of size $n$ having exactly $k$ blocks. They may be arranged in an array having row sums equal to the Catalan number such that the entry in the $n$-th row and $k$-th column is $N_{n, k}$ (sometimes called the Catalan triangle). The Narayana numbers (indeed, a $q$-analogue of them) were first discovered by MacMahon (see Article 495 of [7]) and later rediscovered by Narayana [9]. The $N_{n, k}$ are given by the generating function

$$
N(x, q)=\sum_{n, k \geq 0} N_{n, k} x^{n} q^{k}=\frac{1+x(1-q)-\sqrt{1-2 x(1+q)+x^{2}(1-q)^{2}}}{2 x}
$$

and have the closed form $N_{n, k}=\frac{1}{k}\left(\begin{array}{c}n-1 \\ k-1\end{array}\right)\left(\begin{array}{c}n \\ k-1\end{array}\right)$ if $n, k \geq 1$. For further information on these numbers, see A001263 in [14] as well as the paper by Sulanke [17].

In this paper, we identify eight classes of the partitions of $[n]$ each avoiding a classical pattern of length four and another of length five and each enumerated by the Catalan number $C_{n}$. Furthermore, there are $N_{n, k}$ members of each class having a prescribed number $k$ of blocks. In addition to providing apparently new combinatorial interpretations for the Catalan and Narayana sequences, this addresses specific cases of a general question raised by Goyt [3], for example, regarding the enumeration of classes of partitions avoiding two or more patterns. Analogous results concerning the avoidance of two patterns by a permutation have been given, for example, by Bóna [2] and West [19]. Our main result is the following theorem which we prove as a series of propositions in the next section.

Theorem 1.1. If $n, k \geq 0$, then $p_{n, k}(u, v)=N_{n, k}$ for the following pairs $(u, v)$ :
(1) $(1222,12323)$
(2) $(1222,12332)$
(3) $(1211,12321)$
(4) $(1211,12312)$
(5) $(1121,12231)$
(6) $(1121,12132)$
(7) $(1112,12123)$
(8) $(1112,12213)$.

In particular, we have $p_{n}(u, v)=C_{n}$ for all $n$.

To show this, we give algebraic proofs for cases (1), (3), (5), (6), and (7) and find one-to-one correspondences between cases (1) and (2), (3) and (4), and (7) and (8). To establish (3), (5), (6), and (7), we make use of the kernel method (see [1]) to solve the functional equations that arise once certain parameters have been introduced. Our bijections between cases (3) and (4) and between (7) and (8) are of an algorithmic nature and systematically replace occurrences of 12312 (resp., 12213) with ones of 12321 (resp., 12123) without introducing 1211 (resp., 1112). Furthermore, based on numerical evidence, 
there are no other pattern pairs $u$ and $v$ of lengths four and five such that $p_{n}(u, v)=C_{n}$ for all $n$.

We remark that in proving (3), (5), (6), and (7) above, we make use of auxiliary statistics in two fundamentally different ways which enable us to find a recurrence that we can solve and establish the result. For cases (3) and (5) in section 2.2 below, to find $p_{n, k}(u, v)$, we first refine this number by describing a statistic on $P_{n, k}(u, v)$ and letting $a_{n, k, c}$ denote the number of elements of $P_{n, k}(u, v)$ for which the value of the statistic is $c$. We then write a recurrence for $a_{n, k, c}$, which we are able to solve by the kernel method and thus find $p_{n, k}(u, v)=\sum_{c>0} a_{n, k, c}$. See Zeilberger [21] for a further description and examples of this strategy of refinement in finding an explicit formula for a sequence. For cases (6) and (7) in section 2.3 below, we make use of a different strategy wherein we consider a nested sequence of subsets $\mathcal{S}_{0} \supseteq \mathcal{S}_{1} \supseteq \cdots$ of $P_{n, k}(u, v)$ such that all members of $\mathcal{S}_{i}$ have statistic value at least $i$ for a certain statistic defined on $P_{n, k}(u, v)$. We then are able to find a recurrence for generating functions related to this statistic, which we can solve by the kernel method and thus establish the cardinality of $\mathcal{S}_{0}=P_{n, k}(u, v)$.

\section{Proof of the main result}

\subsection{The cases $\{1222,12323\}$ and $\{1222,12332\}$}

In this section, we consider the cases of avoiding $\{1222,12323\}$ and $\{1222,12332\}$.

Proposition 2.1. We have

$$
\sum_{n, k \geq 0} p_{n, k}(1222,12323) x^{n} q^{k}=N(x, q) .
$$

Hence, for all $n, k \geq 1$, the number of set partitions of $[n]$ with exactly $k$ blocks that avoid both 1222 and 12323 is given by $\frac{1}{k}\left(\begin{array}{c}n-1 \\ k-1\end{array}\right)\left(\begin{array}{c}n \\ k-1\end{array}\right)$, the Narayana number $N_{n, k}$.

Proof. Suppose $\pi=\pi_{1} \pi_{2} \cdots$ is non-empty and avoids $\{1222,12323\}$. Then $\pi$ may be formed by writing a non-empty sequence of the letter 1 directly in front of $\alpha=$ $\alpha_{1} \alpha_{2} \cdots$ and then writing a possibly empty sequence of 1's directly after each letter of $\alpha$, where $\alpha$ is some partition on the letters $\{2,3, \ldots\}$ avoiding $\{111,1212\}$. Let $A(x, q)=\sum_{n, k \geq 0} p_{n, k}(1222,12323) x^{n} q^{k}$ and $B(x, q)=\sum_{n, k \geq 0} p_{n, k}(111,1212) x^{n} q^{k}$. From the foregoing observation, we have

$$
A(x, q)=1+\frac{x q}{1-x} B\left(\frac{x}{1-x}, q\right) .
$$

To compute $B(x, q)$, note that any non-empty partition $\beta$ avoiding $\{111,1212\}$ may be written as either $\beta=1 \beta^{\prime}$ or $\beta=1 \beta^{\prime} 1 \beta^{\prime \prime}$, where $\beta^{\prime}$ and $\beta^{\prime \prime}$ contain no 1's and avoid $\{111,1212\}$. Furthermore, every letter of $\beta^{\prime \prime}$ is greater than every letter of $\beta^{\prime}$ in the second case. Thus $B(x, q)$ satisfies the relation

$$
B(x, q)=1+x q B(x, q)+x^{2} q B^{2}(x, q),
$$


which implies

$$
B(x, q)=\frac{1-x q-\sqrt{1-2 x q+x^{2}\left(q^{2}-4 q\right)}}{2 x^{2} q} .
$$

Substituting (3) into (2) yields the requested formula for $A(x, q)$.

We now show the equivalence of avoiding $\{1222,12323\}$ and $\{1222,12332\}$.

Proposition 2.2. If $n, k \geq 0$, then $p_{n, k}(1222,12323)=p_{n, k}(1222,12332)$.

Proof. Suppose $\pi \in P_{n, k}(1222,12323)$ has exactly $j$ occurrences of the letter 1 , where $n, k \geq 2$. Cover up all of the 1's in $\pi$ and let $\alpha$ denote the remaining letters, which constitute a member of $P_{n-j, k-1}(111,1212)$ (on the letters $\{2,3, \ldots\}$ ). By Corollary 19 in [4], we know that there exists a bijection from $P_{m, i}(1212)$ to $P_{m, i}(1221)$ for all $m$ and $i$ which preserves block sizes, i.e., the number of occurrences of each letter in the canonical form. Thus, it restricts to a bijection between $P_{n-j, k-1}(111,1212)$ and $P_{n-j, k-1}(111,1221)$. Let $\alpha^{\prime}$ denote the corresponding member of $P_{n-j, k-1}(111,1221)$. Replace the letters of $\alpha$ in $\pi$ with those of $\alpha^{\prime}$ (keeping the positions of the 1's fixed). If $\pi^{\prime}$ denotes the resulting partition, then $\pi^{\prime}$ belongs to $P_{n, k}(1222,12332)$ and the mapping $\pi \mapsto \pi^{\prime}$ is a bijection.

\subsection{The cases $\{1211,12321\},\{1121,12231\}$, and $\{1211,12312\}$}

We will first consider the case of avoiding $\{1211,12321\}$. In what follows, we will call a (maximal) sequence of identical consecutive letters a run (of the letter). We will consider forming members of $P_{n, k}(1211,12321)$ by first writing a non-empty sequence of the letter $k$ and then subsequently adding the letters $k-1, k-2, \ldots, 1$.

Definition 2.3. Given a partition $\pi=\pi_{1} \pi_{2} \cdots \pi_{m}$ on the letters $\{2,3, \ldots\}$ and avoiding the patterns 1211 and 12321, let us call the letter $\pi_{i}$ an active site if one may write a single 1 directly to the right of $\pi_{i}$ within the partition $1 \pi$ without creating an occurrence of 12321. All other letters of $\pi$ will be said to be inactive.

Our use of the phrase active site is in accordance with the generating tree methodology described in [19]. Note that no occurrence of 1211 is created since only a single 1 is added after an element greater than or equal to two and also nothing changes if one adds a non-empty run of 1's having arbitrary length at the beginning instead of a single 1 . To illustrate, consider the partition $\pi=2234355546$ on $\{2,3, \ldots\}$ avoiding $\{1211,12321\}$. Note that the first four letters of $\pi$ are active, whereas the last six are inactive (in general, all letters coming after and including the second 1 in the leftmost occurrence of 121 in such a partition are inactive, while those coming before it are active).

Let $\mathcal{A}_{n, k, c}$ denote the set of partitions of length $n$ on the letters $\{2,3, \ldots, k+1\}$ avoiding the patterns 1211 and 12321 and having exactly $c$ active sites. Let $a_{n, k, c}:=\left|\mathcal{A}_{n, k, c}\right|$; note that $p_{n, k}(1211,12321)=\sum_{c=1}^{n} a_{n, k, c}$ for all $n, k \geq 1$ The following lemma provides an explicit recurrence for the array $a_{n, k, c}$, where $n, k, c \geq 1$. 
Lemma 2.4. The numbers $a_{n, k, c}$ satisfy the recurrence

$$
a_{n, k, c}=\sum_{j=1}^{c-1} a_{n-j, k-1, c-j}+\sum_{j=1}^{c-1} \sum_{t=c-j}^{n-j-1} a_{n-j-1, k-1, t}, \quad n \geq k, c \geq 2,
$$

with $a_{n, 1, c}=\delta_{n, c}$ if $1 \leq c \leq n$ and $a_{n, k, c}=0$ if $k>n$, if $c>n$, or if $n \geq 2$ and $c=1$.

Proof. The boundary conditions are readily verified. Note that if $n \geq 2$, then there are at least two active sites in $\pi \in P_{n}$ (the letters used in $\pi$ being $\{2,3, \ldots\}$ ), and if $\pi \in P_{n, 1}$, then all of the letters of $\pi$ correspond to active sites.

If $k, c \geq 2$, we first observe that a member $\pi \in \mathcal{A}_{n, k, c}$ may be obtained by writing a run of 1 's of length $j$ just before a member $\alpha \in \mathcal{A}_{n-j, k-1, c-j}$ for some $j, 1 \leq j \leq c-1$, and then adding one to each letter of the resulting partition. Note that all of the active sites in $\alpha$ remain active in $\pi$ and that there are $j$ additional active sites created corresponding to the positions directly following the $j$ added 1's. Thus, there are $\sum_{j=1}^{c-1} a_{n-j, k-1, c-j}$ possible members of $\mathcal{A}_{n, k, c}$ in this case.

Alternatively, one may add the 1's in the procedure described in the previous paragraph as two separate runs, the second of which has length one. Note that this is the only other option since we are to avoid 1211. Suppose one were to write a sequence consisting of $j$ 1's just before $\beta \in \mathcal{A}_{n-j-1, k-1, t}$, write a single 1 just after the $(c-j)$-th active site of $\beta$ from the left (assuming $c-j \leq t$ and $1 \leq j \leq c-1$ ), and then add one to each letter. The resulting partition $\pi$ belongs to $\mathcal{A}_{n, k, c}$. To see this, note that all of the active sites in $\beta$ to the left of (and including) the $(c-j)$-th one remain active in $\pi$, with the $j 2$ 's in the first run also active. On the other hand, the active sites of $\beta$ to the right of $(c-j)$-th one become inactive in $\pi$, with the final 2 in $\pi$ also inactive. Furthermore, no other active sites are lost or created in the transition from $\beta$ to $\pi$. T hus, there are $\sum_{j=1}^{c-1} \sum_{t=c-j}^{n-j-1} a_{n-j-1, k-1, t}$ members of $\mathcal{A}_{n, k, c}$ having exactly $j+12$ 's occurring as two runs for some $j$. Combining this case with the prior one yields (4).

Using recurrence (4), one can find $p_{n, k}(1211,12321)$.

Proposition 2.5. We have

$$
\sum_{n, k \geq 0} p_{n, k}(1211,12321) x^{n} q^{k}=N(x, q)
$$

Proof. Define $A_{n, k}(v)=\sum_{c=1}^{n} a_{n, k, c} v^{c-1}$. Multiplying recurrence (4) by $v^{c-1}$ and summing 
over $1 \leq c \leq n$ (note that (4) also holds for $c=1$ ), we obtain

$$
\begin{aligned}
A_{n, k}(v) & =\sum_{j=1}^{n-2} \sum_{i=j}^{n-2}\left(v^{i} \sum_{t=i-j+1}^{n-j-1} a_{n-1-j, k-1, t}\right)+\sum_{j=1}^{n-1} \sum_{i=1}^{j} a_{j, k-1, i} v^{n+i-j-1} \\
& =\sum_{j=1}^{n-2} \sum_{i=1}^{n-j-1} \frac{v^{j}\left(1-v^{i}\right)}{1-v} a_{n-1-j, k-1, i}+\sum_{j=1}^{n-1} v^{j} A_{n-j, k-1}(v) \\
& =\sum_{j=1}^{n-2} \frac{v^{j}}{1-v}\left(A_{n-1-j, k-1}(1)-v A_{n-1-j, k-1}(v)\right)+\sum_{j=1}^{n-1} v^{j} A_{n-j, k-1}(v) \\
& =\sum_{j=1}^{n-k} \frac{v^{j}}{1-v}\left(A_{n-1-j, k-1}(1)-v A_{n-1-j, k-1}(v)\right)+\sum_{j=1}^{n-k+1} v^{j} A_{n-j, k-1}(v),
\end{aligned}
$$

where $n, k \geq 2$, since $A_{m, j}(v)=0$ if $j>m$. Now define $A_{k}(x ; v)=\sum_{n \geq k} A_{n, k}(v) x^{n}$. Then the above recurrence gives

$$
\begin{aligned}
A_{k}(x ; v)= & \sum_{n \geq k}\left(x^{n} \sum_{j=1}^{n-k} \frac{v^{j}}{1-v}\left(A_{n-1-j, k-1}(1)-v A_{n-1-j, k-1}(v)\right)\right) \\
& +\sum_{n \geq k}\left(x^{n} \sum_{j=1}^{n-k+1} v^{j} A_{n-j, k-1}(v)\right) \\
= & \frac{x^{2} v}{(1-v)(1-x v)} \sum_{j \geq k-1}\left(A_{j, k-1}(1) x^{j}-v A_{j, k-1}(v) x^{j}\right)+\frac{x v}{1-x v} \sum_{j \geq k-1} A_{j, k-1}(v) x^{j} \\
= & \frac{x^{2} v}{(1-v)(1-x v)}\left(A_{k-1}(x ; 1)-v A_{k-1}(x ; v)\right)+\frac{x v}{1-x v} A_{k-1}(x ; v), \quad k \geq 2 .
\end{aligned}
$$

Define $A(x, q ; v)=\sum_{k \geq 1} A_{k}(x ; v) q^{k}$. Multiplying the above recurrence by $q^{k}$, summing over all $k \geq 2$, and noting the initial condition $A_{1}(x ; v)=\frac{x}{1-x v}$, we obtain

$$
A(x, q ; v)=\frac{x q}{1-x v}+\frac{x^{2} q v}{(1-v)(1-x v)}(A(x, q ; 1)-v A(x, q ; v))+\frac{x q v}{1-x v} A(x, q ; v),
$$

which is equivalent to

$$
\left(1-\frac{x q v}{1-x v}+\frac{x^{2} q v^{2}}{(1-v)(1-x v)}\right) A(x, q ; v)=\frac{x q}{1-x v}+\frac{x^{2} q v}{(1-v)(1-x v)} A(x, q ; 1) .
$$

This type of functional equation may be solved systematically using the kernel method (see [1]). If we set the coefficient of $A(x, q ; v)$ in (6) equal to zero, and solve for $v=v_{0}$ in terms of $x$ and $q$, we obtain

$$
v_{0}=\frac{1+x(1+q)-\sqrt{1-2 x(1+q)+x^{2}(1-q)^{2}}}{2 x(1+q+x q)} .
$$


Note that of the two possible values of $v_{0}$, only this one yields a power series in $x$ and $q$. Substituting $v=v_{0}$ into (6) gives

$$
\begin{aligned}
\sum_{n, k \geq 1} p_{n, k}(1211,12321) x^{n} q^{k} & =A(x, q ; 1)=\frac{v_{0}-1}{x v_{0}} \\
& =\frac{1-x(1+q)-\sqrt{1-2 x(1+q)+x^{2}(1-q)^{2}}}{2 x},
\end{aligned}
$$

as required.

Using (6) and the expression for $A(x, q ; 1)$, one can find $A(x, q ; v)$ and recover the coefficient $a_{n, k, c}$. We next consider the case of avoiding $\{1121,12231\}$. We start with a similar definition for active site.

Definition 2.6. Given a partition $\pi=\pi_{1} \pi_{2} \cdots \pi_{m}$ on the letters $\{2,3, \ldots\}$ and avoiding the patterns 1121 and 12231, let us call the letter $\pi_{i}$ an active site if one may write a non-empty run of 1 's directly to the right of $\pi_{i}$ within the partition $1 \pi$ without creating an occurrence of 12231. All other letters of $\pi$ will be said to be inactive.

For example, in the partition $\pi=2344225336$ on $\{2,3, \ldots\}$ avoiding $\{1121,12231\}$, the first six letters of $\pi$ are active and no others (note that the 5 corresponds to the 2 in the leftmost occurrence of 112 in $\pi$, so all letters after and including it are inactive). In the definition above, only a single run of 1's is to be inserted into the partition $1 \pi$, whence no occurrences of 1121 are created. Let $\mathcal{B}_{n, k, c}$ denote the set of partitions of length $n$ on the letters $\{2,3, \ldots, k+1\}$ avoiding the patterns 1121 and 12231 and having exactly $c$ active sites. Let $b_{n, k, c}:=\left|\mathcal{B}_{n, k, c}\right|$; note that $p_{n, k}(1121,12231)=\sum_{c=1}^{n} b_{n, k, c}$ for all $n, k \geq 1$ The following lemma provides an explicit recurrence for the array $b_{n, k, c}$, where $n, k, c \geq 1$.

Lemma 2.7. The numbers $b_{n, k, c}$ satisfy the recurrence

$$
b_{n, k, c}=b_{n-1, k-1, c-1}+\sum_{t=1}^{n-c} b_{n-c, k-1, t}+\sum_{j=2}^{c-1} \sum_{t=c-j}^{n-j} b_{n-j, k-1, t}, \quad n \geq k, c \geq 2,
$$

with $b_{n, 1, c}=\delta_{n, c}$ if $1 \leq c \leq n$ and $b_{n, k, c}=0$ if $k>n$, if $c>n$, or if $n \geq 2$ and $c=1$.

Proof. The boundary conditions are readily verified, so assume $k, c \geq 2$. First suppose that there is only one run of 2's in $\pi \in \mathcal{B}_{n, k, c}$ and that its length is $j$. Then either $j=1$ or $j=c$. For if $j \geq 2$, then the first $j$ positions of $\pi$ are all active and no others, which implies $j=c$. If $j=1$, then there are $b_{n-1, k-1, c-1}$ possible members of $\mathcal{B}_{n, k, c}$, while if $j=c$, there are $\sum_{t=1}^{n-c} b_{n-c, k-1, t}=p_{n-c, k-1}(1121,12231)$ possible members.

Alternatively, one may add the 1's as two separate runs, the first of which has length one. Note that this is the only other option since we are to avoid 1121. Suppose we first write a single 1 just before $\beta \in \mathcal{B}_{n-j, k-1, t}$, next write a run of $j-11$ 's just after the $(c-j$ )-th active site of $\beta$ from the left (assuming $c-j \leq t$ and $2 \leq j \leq c-1$ ), and then add one to each letter. Similar reasoning as in the proof of Lemma 2.4 above then shows 
that the resulting partition $\pi$ belongs to $\mathcal{B}_{n, k, c}$ and that the number of partitions formed in this manner is $\sum_{j=2}^{c-1} \sum_{t=c-j}^{n-j} b_{n-j, k-1, t}$. Combining this case with the prior two yields $(7)$.

Proposition 2.8. We have

$$
\sum_{n, k \geq 0} p_{n, k}(1121,12231) x^{n} q^{k}=N(x, q)
$$

Proof. Define $B_{n, k}(v)=\sum_{j=1}^{n} b_{n, k, c} v^{c-1}$. Multiplying recurrence (7) by $v^{c-1}$ and summing over $2 \leq c \leq n$, we obtain for $n, k \geq 2$,

$$
B_{n, k}(v)=v B_{n-1, k-1}(v)+\sum_{j=1}^{n-k} v^{j} B_{n-1-j, k-1}(1)+\sum_{j=2}^{n-k+1} \frac{v^{j}}{1-v}\left(B_{n-j, k-1}(1)-v B_{n-j, k-1}(v)\right) .
$$

Now define $B_{k}(x ; v)=\sum_{n \geq k} B_{n, k}(v) x^{n}$. Then the above recurrence gives for $k \geq 2$,

$$
\begin{aligned}
B_{k}(x ; v)= & x v B_{k-1}(x ; v)+\sum_{n \geq k-1} \frac{x^{n+2} v}{1-x v} B_{n, k-1}(1) \\
& +\sum_{n \geq k-1} \frac{x^{n+2} v^{2}}{(1-v)(1-x v)}\left(B_{n, k-1}(1)-v B_{n, k-1}(v)\right) \\
= & x v B_{k-1}(x ; v)+\frac{x^{2} v}{1-x v} B_{k-1}(x ; 1)+\frac{x^{2} v^{2}}{(1-v)(1-x v)}\left(B_{k-1}(x ; 1)-v B_{k-1}(x ; v)\right) .
\end{aligned}
$$

Define $B(x, q ; v)=\sum_{k>1} B_{k}(x ; v) q^{k}$. Multiplying the above recurrence by $q^{k}$, summing over all $k \geq 2$, and noting the initial condition $B_{1}(x ; v)=\frac{x}{1-x v}$, we obtain

$$
\begin{aligned}
B(x, q ; v)= & \frac{x q}{1-x v}+x q v B(x, q ; v)+\frac{x^{2} q v^{2}}{(1-v)(1-x v)}(B(x, q ; 1)-v B(x, q ; v)) \\
& +\frac{x^{2} q v}{1-x v} B(x, q ; 1),
\end{aligned}
$$

which is equivalent to

$$
\left(1-x q v+\frac{x^{2} q v^{3}}{(1-v)(1-x v)}\right) B(x, q ; v)=\frac{x q}{1-x v}+\frac{x^{2} q v}{(1-v)(1-x v)} B(x, q ; 1) .
$$

We again use the kernel method and substitute $v=v_{0}=\frac{1+x(1+q)-\sqrt{1-2 x(1+q)+x^{2}(1-q)^{2}}}{2 x(1+q+x q)}$ into the last equation to obtain

$$
B(x, q ; 1)=\frac{1-x(1+q)-\sqrt{1-2 x(1+q)+x^{2}(1-q)^{2}}}{2 x},
$$

as required. 
Proposition 2.9. If $n, k \geq 0$, then $p_{n, k}(1211,12321)=p_{n, k}(1211,12312)$.

Proof. We will define an explicit bijection $f$ between the sets $A=P_{n, k}(1211,12321)$ and $B=P_{n, k}(1211,12312)$, assuming $k \geq 3$. Let $\pi \in A$. We will first describe a sequence of partitions $\pi_{2}, \pi_{3}, \ldots, \pi_{k}$, where $\pi_{2}=\pi$ and $\pi_{k}$ belongs to $B$. To do so, first consider the leftmost occurrence of the letter 3 in $\pi_{2}$. Since $\pi_{2}$ avoids both 1211 and 12321, there can be at most one 1 and one 2 occurring after this 3, with the 2 coming later than the 1 if both occur. We define the partition $\pi_{3}$ as follows. If both a 1 and a 2 occur after the leftmost occurrence of the letter 3 in $\pi_{2}$, then we switch the positions of this 1 and 2 to obtain $\pi_{3}$. Otherwise, we let $\pi_{3}=\pi_{2}$.

Now consider the position of the leftmost 4 in $\pi_{3}$. Any members of [3] occurring after this 4 can occur at most once (note that no 3 is moved in the first step). Within the subsequence of $\pi_{3}$ comprising these members of [3], cyclicly shift the 3 , if it occurs, from the last to the first position to obtain $\pi_{4}$; otherwise, let $\pi_{4}=\pi_{3}$. In general, for each $m, 3 \leq m \leq k$, starting with $m=3$, rewrite any elements of [ $m-1]$ occurring after the leftmost $m$ in descending order, if necessary, to obtain the partition $\pi_{m}$ (by shifting the $m-1$, if it occurs, to the first position among these elements); otherwise, let $\pi_{m}=\pi_{m-1}$. Consider the resulting sequence of partitions $\pi_{2}=\pi, \pi_{3}, \ldots, \pi_{k}$, and let $f(\pi)=\pi_{k}$.

Note that $f$ preserves the number of blocks and is seen to be a bijection, its inverse obtained by reversing each of the steps described, starting with the last. To show that $f(\pi) \in B$, note first that any members of $[i-1]$ coming after the leftmost $i$ in $\pi_{i}$ occur singly in descending order. Note further that in the step going from $\pi_{i}$ to $\pi_{i+1}$, the relative order of the elements of $[i-1]$ following the leftmost $i$ does not change as there is only possibly a cyclic shift between the letter $i$ and some of the members of $[i-1]$ which occurs only if there is an $i$ coming after the leftmost $i+1$ (note that the letters in all of the other positions of $\pi_{i}$ are unchanged in this step). Likewise, the members of $[i-1]$ coming after the leftmost $i$ occur singly in descending order in the partition $\pi_{j}$ for each $j>i$ (in fact, this subset of $[i-1]$ is the same in $\pi_{j}$ for all $j>i$ ). Thus, for each letter $i \geq 2$, it is not possible for $i$ to correspond to the 2 in an occurrence of 1211 in $f(\pi)$ nor is it possible for a letter $i \geq 3$ to correspond to the 3 in an occurrence of 12312. This implies $f(\pi) \in B$, as desired.

Below is an example of the bijection $f$ when $n=15$ and $k=7$ :

$$
\begin{aligned}
& \pi=\pi_{2}=123142553647567 \rightarrow \pi_{3}=123241553647567 \rightarrow \pi_{4}=123243551647567 \rightarrow \\
& \pi_{5}=123243554617567 \rightarrow \pi_{6}=123243554657167 \rightarrow \pi_{7}=123243554657617=f(\pi) .
\end{aligned}
$$

\subsection{The cases $\{1121,12132\},\{1112,12123\}$, and $\{1112,12213\}$}

We first consider the case of avoiding $\{1121,12132\}$. To do so, we introduce the auxiliary generating functions $G_{k}=G_{k}(x), k \geq 1$, for the number of partitions of $P_{n}(1121,12132)$ of the form

$$
\pi=12 \cdots k \pi^{(k)} k \pi^{(k-1)}(k-1) \pi^{(k-2)} \cdots 2 \pi^{(1)} 1 \pi^{(0)}
$$


where the $\pi^{(i)}, 0 \leq i \leq k$, are possibly empty and contain only letters in $\{k+1, k+2, \ldots\}$. Note that the subset of $P_{n}(1121,12132)$ counted by the coefficient of $x^{n}$ in $G_{j}$ is contained within the subset counted by the coefficient of $x^{n}$ in $G_{i}$ if $i<j$, with $G_{0}$ enumerating all members of $P_{n}(1121,12132)$, by definition. It is possible to give a recurrence for the $G_{k}$.

Lemma 2.10. If $k \geq 1$, then

$$
G_{k}=x^{2} G_{k-1}+x G_{k}+\frac{1}{1-x} G_{k+1}+\sum_{m=0}^{k-1} \frac{x^{2(k-m)}}{(1-x)^{k+1-m}} G_{m+1},
$$

with $G_{0}=\sum_{n \geq 0} p_{n}(1121,12132) x^{n}$.

Proof. Let $H_{k}=H_{k}(x)$ be the generating function for the same partitions counted by $G_{k}$, but with the added condition $\pi^{(k)} \neq \varnothing$. From the definitions, we then have

$$
G_{k}=x^{2} G_{k-1}+H_{k}, \quad k \geq 1,
$$

since, if $\pi^{(k)}$ is empty, we may simply delete the two (consecutive) occurrences of the letter $k$ as they can play no role in forming a possible 1121 or 12132 subsequence.

Note that in partitions counted by $H_{k}$, the word $\pi^{(k)}$ must begin with the letter $k+1$. We now consider the following cases concerning the last occurrence of the letter $k+1$ in a partition $\pi$ counted by $H_{k}$ :

1. Exactly one $k+1$ occurs in $\pi$.

2. There are at least two occurrences of $k+1$ in $\pi$, with the last occurrence in $\pi^{(m)}$ for some $m, 0 \leq m \leq k-1$.

3. There are at least two occurrences of $k+1$ in $\pi$, with the last one in $\pi^{(k)}$.

In the first case, we may safely delete the $k+1$ from $\pi$ without affecting the enumeration since it is extraneous concerning possible occurrences of 1121 and 12132, which implies that the contribution in this case is $x G_{k}$. In the second case, observe that there can be no letters $c$ greater than $k+1$ between the second $k$ and the last occurrence of the letter $k+1$, for otherwise there would be an occurrence of 12132 involving $k, k+1$, and $c$. Also in this case, if a second $k+1$ occurs in $\pi^{(k)}$, then there can be no letter $d$ greater than $k+1$ coming after it in $\pi^{(k)}$, for otherwise there would be an occurrence of 1121 with $k+1$ and $d$. Putting these observations together, we see that $\pi$ must have the form

$$
\begin{aligned}
\pi= & 12 \cdots k(k+1) \alpha^{(k)}(k+1) \cdots(k+1) k(k+1) \cdots(k+1)(k-1) \cdots(m+1) \\
& (k+1) \cdots(k+1) \alpha^{(m)} m \alpha^{(m-1)}(m-1) \cdots \alpha^{(1)} 1 \alpha^{(0)},
\end{aligned}
$$

where $\alpha^{(0)}, \alpha^{(1)}, \ldots, \alpha^{(m)}$ and $\alpha^{(k)}$ are possibly empty and contain only letters in $\{k+$ $2, k+3, \ldots\}$ and where all but the last run of the letter $k+1$ are possibly empty. Note that one may pinch off both copies of the letters $m+1, m+2, \ldots, k-1$ as well as all of 
the $k+1$ 's since they are extraneous concerning a possible occurrence of 1121 or 12132 . This leaves partitions of the form

$$
\pi=12 \cdots m k \alpha^{(k)} k \alpha^{(m)} m \alpha^{(m-1)}(m-1) \cdots \alpha^{(1)} 1 \alpha^{(0)}
$$

to consider concerning the avoidance of 1121 and 12132, which are clearly enumerated by $G_{m+1}$. Thus, for each $m, 0 \leq m \leq k-1$, partitions of the form described in the second case are enumerated by the generating function $\frac{x^{2(k-m)}}{(1-x)^{k-m}} G_{m+1}$, upon restoring the two copies of the letters $m+1, \ldots, k-1$ and $k+1$ as well as any other $k+1$ 's occurring in the runs.

In the third case above, we see that partitions must have the form

$$
\pi=12 \cdots k(k+1) \alpha^{(k+1)}(k+1) \cdots(k+1) \alpha^{(k)} k \alpha^{(k-1)}(k-1) \alpha^{(k-2)} \cdots 1 \alpha^{(0)},
$$

where the run of the letter $k+1$ is non-empty and the $\alpha^{(i)}$ are possibly empty. Pinching off any $k+1$ 's occurring in this run past the first one, we see that the partitions in this case contribute $\frac{1}{1-x} G_{k+1}$. Combining the three cases yields

$$
H_{k}=x G_{k}+\sum_{m=0}^{k-1} \frac{x^{2(k-m)}}{(1-x)^{k-m+1}} G_{m+1}+\frac{1}{1-x} G_{k+1}, \quad k \geq 1 .
$$

Substituting (11) into (10) gives (9).

Proposition 2.11. We have

$$
\sum_{n \geq 0} p_{n}(1121,12132) x^{n}=C(x)
$$

Proof. First note that a partition avoiding $\{1121,12132\}$ may either be empty, have exactly one occurrence of 1 , or be of the form $\pi=1 \pi^{\prime} 1 \cdots 1 \pi^{\prime \prime}$, where the run of 1 's in the middle is non-empty and $\pi^{\prime}$ and $\pi^{\prime \prime}$ contain no 1's and are possibly empty. This implies the relation

$$
G_{0}=1+x G_{0}+\frac{1}{1-x} G_{1}
$$

or $G_{1}=(1-x)^{2} G_{0}-(1-x)$. Now let $G(x, t)=\sum_{k \geq 0} G_{k}(x) t^{k} ;$ note that $G(x, 0)=$ $G_{0}(x)=\sum_{n \geq 0} p_{n}(1121,12132) x^{n}$. Multiplying (9) by $t^{k}$ and summing over $k \geq 1$ yields

$$
\begin{aligned}
G(x, t)-G(x, 0)= & x^{2} t G(x, t)+x(G(x, t)-G(x, 0)) \\
& +\frac{1}{(1-x) t}\left(G(x, t)-G_{1}(x) t-G(x, 0)\right) \\
& +\sum_{k \geq 1} t^{k} \sum_{m=0}^{k-1} \frac{x^{2(k-m)}}{(1-x)^{k-m+1}} G_{m+1}(x) \\
= & \left(x^{2} t+x+\frac{1}{(1-x) t}\right) G(x, t)-\left(1+\frac{1}{(1-x) t}\right) G(x, 0)+1 \\
& +\frac{x^{2}}{(1-x)\left(1-x-x^{2} t\right)}(G(x, t)-G(x, 0)),
\end{aligned}
$$


which may be rewritten as

$$
\frac{\left(1-x^{2} t\right)\left(1-t+2 x t+x^{2} t^{2}\right)}{\left(1-x-x^{2} t\right) t} G(x, t)=\frac{1}{\left(1-x-x^{2} t\right) t} G(x, 0)-1 .
$$

Setting the coefficient of $G(x, t)$ equal to zero in (13), and solving for $t$, we obtain the three roots

$$
t_{0}=\frac{1}{x^{2}}, \frac{1-2 x+\sqrt{1-4 x}}{2 x^{2}}, \frac{1-2 x-\sqrt{1-4 x}}{2 x^{2}},
$$

only the last of which is permissible since $t_{0}=t(x)$ must be a power series in $x$. Thus, we have

$$
\sum_{n \geq 0} p_{n}(1121,12132) x^{n}=G(x, 0)=\left(1-x-x^{2} t_{0}\right) t_{0}=\frac{1-\sqrt{1-4 x}}{2 x},
$$

as required.

Remark 2.12. One may also add a parameter $q$ marking the number of blocks and consider the further generating function $G_{k}(x, q)$, where $G_{k}(x, 1)=G_{k}(x)$. Then the reasoning used in the proof of Lemma 2.10 shows that the $G_{k}(x, q)$ satisfy the following recurrence for $k \geq 1$ :

$$
G_{k}(x, q)=x^{2} q G_{k-1}(x, q)+x q G_{k}(x, q)+\frac{1}{1-x} G_{k+1}(x, q)+\sum_{m=0}^{k-1} \frac{x^{2(k-m)} q^{k-m}}{(1-x)^{k-m+1}} G_{m+1}(x, q) .
$$

Proceeding in a similar manner, one may obtain

$$
\sum_{n, k \geq 0} p_{n, k}(1121,12132) x^{n} q^{k}=N(x, q)
$$

which implies $p_{n, k}(1121,12132)$ is the Narayana number.

We next consider the case of avoiding $\{1112,12123\}$. We will use the same pyramidal structure as before. Let $U_{k}=U_{k}(x), k \geq 1$, be the generating function for the number of partitions of $P_{n}(1112,12123)$ of the form

$$
\pi=12 \cdots k \pi^{(k)} k \pi^{(k-1)}(k-1) \pi^{(k-2)} \cdots 2 \pi^{(1)} 1 \pi^{(0)},
$$

where the $\pi^{(i)}, 0 \leq i \leq k$, are possibly empty and contain only letters in $\{k+1, k+2, \ldots\}$. We now give a recurrence for the $U_{k}$.

Lemma 2.13. If $k \geq 1$, then

$$
U_{k}=x^{2} U_{k-1}+x U_{k}+U_{k+1}+\sum_{m=0}^{k-1} \frac{x^{2 m+2}}{(1-x)^{m+1}} U_{k-m}+\sum_{m=0}^{k} \frac{x^{2 m+1}}{(1-x)^{m+1}} U_{k+1-m},
$$

with $U_{0}=\sum_{n \geq 0} p_{n}(1112,12123) x^{n}$. 
Proof. Let $V_{k}=V_{k}(x)$ be the generating function for the same partitions counted by $U_{k}$, but with the added condition $\pi^{(k)} \neq \varnothing$. From the definitions, we then have

$$
U_{k}=x^{2} U_{k-1}+V_{k}, \quad k \geq 1 .
$$

Note that in partitions counted by $V_{k}$, the word $\pi^{(k)}$ must begin with the letter $k+1$. We now consider the following cases concerning a possible second occurrence of the letter $k+1$ in a partition $\pi$ counted by $V_{k}$ :

1. Exactly one $k+1$ occurs in $\pi$.

2. There are at least two occurrences of $k+1$ in $\pi$, with the second occurrence in $\pi^{(m)}$ for some $m, 0 \leq m \leq k-1$.

3. There is a second $k+1$ in $\pi^{(k)}$.

In the first case, we may safely delete the $k+1$ from $\pi$ without affecting the enumeration since it is extraneous concerning possible occurrences of 1112 and 12123, which implies that the contribution in this case is $x U_{k}$. In the second case, observe that there can be no letters greater than $k+1$ to the right of the second $k+1$, for otherwise there would be an occurrence of 12123 . Thus, $\pi$ must have the form

$$
\begin{aligned}
\pi= & 12 \cdots k(k+1) \alpha^{(k)} k \alpha^{(k-1)}(k-1) \cdots \alpha^{(m+1)}(m+1) \alpha^{(m)}(k+1) \cdots(k+1) \\
& m(k+1) \cdots(k+1)(m-1) \cdots 2(k+1) \cdots(k+1) 1(k+1) \cdots(k+1),
\end{aligned}
$$

where $\alpha^{(m)}, \alpha^{(m-1)}, \ldots, \alpha^{(k)}$ are possibly empty and contain only letters in $\{k+2, k+3, \ldots\}$ and where all but the first run of the letter $k+1$ are possibly empty. Note that one may expunge both copies of the letters $1,2, \ldots, m$ as well as all of the $k+1$ 's since they are extraneous concerning a possible occurrence of 1112 or 12123 . Thus, for each $m$, partitions of the form described in the second case are enumerated by the generating function $\frac{x^{2 m+2}}{(1-x)^{m+1}} U_{k-m}, 0 \leq m \leq k-1$. Combining the three cases implies

$$
V_{k}=x U_{k}+\sum_{m=0}^{k-1} \frac{x^{2 m+2}}{(1-x)^{m+1}} U_{k-m}+W_{k}, \quad k \geq 1,
$$

where $W_{k}$ is the generating function counting the partitions of the form described in the third case above.

To compute $W_{k}$, consider cases on whether there are two or more occurrences of the letter $k+1$ and, if there are more, on the occurrence of the third $k+1$. If there are exactly two $k+1$ 's, then the contribution is $U_{k+1}$. On the other hand, if the third $k+1$ occurs in $\pi^{(m)}$, where $0 \leq m \leq k$, then reasoning similar to that used in the prior paragraph shows that the contribution is $\frac{x^{2 m+1}}{(1-x)^{m+1}} U_{k+1-m}$. Thus, $W_{k}=U_{k+1}+\sum_{m=0}^{k} \frac{x^{2 m+1}}{(1-x)^{m+1}} U_{k+1-m}$. Recurrence (14) now follows from (15) and (16). 
Note that the recurrence for $G_{k}$ in (9) above may be rewritten as

$$
\begin{aligned}
G_{k} & =x^{2} G_{k-1}+x G_{k}+\frac{1}{1-x} G_{k+1}+\left(1+\frac{x}{1-x}\right) \sum_{m=0}^{k-1} \frac{x^{2(k-m)}}{(1-x)^{k-m}} G_{m+1} \\
& =x^{2} G_{k-1}+x G_{k}+G_{k+1}+\sum_{m=0}^{k-1} \frac{x^{2 m+2}}{(1-x)^{m+1}} G_{k-m}+\sum_{m=0}^{k} \frac{x^{2 m+1}}{(1-x)^{m+1}} G_{k+1-m}
\end{aligned}
$$

which is the same as the recurrence for $U_{k}$ in (14). This implies, by the kernel method, that $U_{0}=G_{0}$, which yields the following result.

Proposition 2.14. If $n \geq 0$, then $p_{n}(1112,12123)=C_{n}$.

Remark 2.15. Upon adding an additional parameter $q$ that marks the number of blocks, one can show further that $p_{n, k}(1112,12123)=N_{n, k}$. It is also possible to construct a direct bijection preserving the number of blocks between the partitions counted by $G_{j}$ and those counted by $U_{j}$ for all $j \geq 1$, which implies $p_{n, k}(1121,12132)=p_{n, k}(1112,12123)$.

Finally, we come to the case of avoiding $\{1112,12213\}$. For this, we will define an explicit bijection between $P_{n}(1112,12123)$ and $P_{n}(1112,12213)$ which preserves the number of blocks. We will need the following two definitions, the first of which is directly analogous to one given for permutations.

Definition 2.16. Given a partition $\pi=\pi_{1} \pi_{2} \cdots \pi_{n} \in P_{n}$, the position $\pi_{i}$ will be called a right-to-left maximum of $\pi(\mathrm{rlm})$ if there exists no index $j>i$ such that $\pi_{j} \geq \pi_{i}$; by definition, the final letter $\pi_{n}$ of $\pi$ is always an $\mathrm{rlm}$.

For example, if $\pi=123243423322 \in P_{12}$, then the letters in the seventh, tenth, and twelfth positions correspond to rlm's.

Definition 2.17. If $\pi \in P_{n, k}$ and $i \in[k]$, then we will denote by $\pi \cap[i]$ the word obtained by replacing each letter of $\pi$ greater than $i$ with the symbol $x$.

For example, if $\pi=1232442535 \in P_{10,5}$ and $i=3$, then $\pi \cap[i]=1232 x x 2 x 3 x$. Note that $\pi \cap[i]$ is itself a partition having $i$ blocks when one considers only the positions occupied by letters. In the proof which follows, we will often perform operations on the partition $\pi \cap[i]$ and then restore the letters greater than $i$ to their original positions.

Proposition 2.18. If $n, k \geq 0$, then $p_{n, k}(1112,12123)=p_{n, k}(1112,12213)$.

Proof. We will define an explicit bijection between the sets $A=P_{n, k}(1112,12123)$ and $B=P_{n, k}(1112,12213)$. Suppose $\pi=\pi_{1} \pi_{2} \cdots \pi_{n} \in A$ has exactly $t$ rlm's. Let $m_{1}<m_{2}<$ $\cdots<m_{t}$ denote the set of letters corresponding to the rlm's of $\pi$ (note $m_{1}=\pi_{n}$ and $\left.m_{t}=k\right)$. Let $a_{i}$ denote the index of the position of the rightmost occurrence of $m_{i}$ within $\pi$ for each $i \in[t]$ (note $a_{1}>a_{2}>\cdots>a_{t}$, with $a_{1}=n$ and $a_{t}$ equal the index of the rightmost $k$ in $\pi$ ). For example, if $n=12, k=4$, and $\pi=123324414332 \in A$, then $t=3$, with $m_{1}=2, m_{2}=3, m_{3}=4$ and $a_{1}=12, a_{2}=11, a_{3}=9$. 
Let $\pi_{0}=\pi$ and suppose we form a partition $\pi_{1}$ from $\pi_{0}$ in the following manner. First, move all of the letters of $m_{1}-1$ within $\pi_{0} \cap\left[m_{1}-1\right]$ except the first, which remains fixed in its position, to the very end (so as to remove any occurrences of 1221 in which the 2 corresponds to $\left.m_{1}-1\right)$. Once this operation is done, put back the letters belonging to $\left\{m_{1}, m_{1}+1, \ldots, k\right\}$ (which were covered by $x$ 's) and let $\pi_{0}^{(1)}$ denote the resulting partition. Then repeat with $\pi_{0}^{(1)} \cap\left[m_{1}-2\right]$, moving all of the $\left(m_{1}-2\right)$ 's to the very end, except for the first, calling the resulting partition $\pi_{0}^{(2)}$. Subsequently repeat this for $m_{1}-3, m_{1}-4, \ldots, 1$ to obtain a sequence of partitions $\pi_{0}^{(0)}=\pi_{0}, \pi_{0}^{(1)}, \ldots, \pi_{0}^{(m-1)}$ (all of which have length $n$ ). Let $\pi_{1}=\pi_{0}^{(m-1)}$.

Let $\pi_{1}^{\prime}$ denote the partition obtained by considering only the first $a_{2}$ positions of $\pi_{1}$. Now repeat the procedure described in the previous paragraph starting with $\pi_{1}^{\prime}$, this time using the letters $m_{2}-1, m_{2}-2, \ldots, m_{1}$. That is, first move all $\left(m_{2}-1\right)$ 's, except the first, to the end of $\pi_{1}^{\prime} \cap\left[m_{2}-1\right]$. Then repeat this with $m_{2}-2$ on the resulting partition intersected with $\left[m_{2}-2\right]$, etc., until one considers $m_{1}$. Let $\pi_{2}$ denote the partition of length $n$ obtained after one performs the final step described with $m_{1}$, puts back all of the letters belonging to $\left\{m_{1}+1, m_{1}+2, \ldots, k\right\}$ (which are covered by $x$ 's at this point), and also puts back all of the letters coming after position $a_{2}$. Let $\pi_{2}^{\prime}$ denote the first $a_{3}$ positions of $\pi_{2}$, and repeat the process with $\pi_{2}^{\prime}$ and the letters $m_{3}-1, m_{3}-2, \ldots, m_{2}$ to obtain the partition $\pi_{3}$.

Now repeat the above procedure $t$ times (in the last step, one moves the letters $m_{t}-1=$ $\left.k-1, m_{t}-2, \ldots, m_{t-1}\right)$, which yields a sequence of partitions $\pi_{0}=\pi, \pi_{1}, \pi_{2}, \ldots, \pi_{t}$. Let $f(\pi)=\pi_{t}$. Since the process is seen to preserve the positions of rlm's, its inverse can be obtained by composing the inverses of the individual steps in reverse order. Note further that the mapping $f$ preserves the number of blocks in a partition (in fact, $\pi_{i} \in P_{n, k}$ for all $i \in[t])$.

We now show $f(\pi) \in B$. First note that there is an occurrence of 12213 in a partition $\pi$ if and only if there is an occurrence of 12213 in which the 3 corresponds to an rlm, and, likewise, one may assume that the 2 in a possible occurrence of 1112 also corresponds to an rlm. Observe further that once the letters of the element $\ell \in[k-1]$ are moved in the procedure described, then there can be no occurrences of 12213 arising in later steps where the 2 corresponds to $\ell$. To see this, suppose letters of $j \in[k-1]$ are moved in some later step. If $j>\ell$, then no 12213 can arise in which the 2 corresponds to $\ell$ since the relative order of the letters belonging to $[\ell]$ does not change in this case, and if $j<\ell$, then no such 12213 can arise since the positions of the letters $\ell$ relative to those in $[\ell-1]$ do not change in later steps. Similarly, no 1112 can arise since one may verify that the number of times any $u \in[k-1]$ appears to the left of $v$ remains fixed throughout the process, where $v$ is the smallest rlm with $v>u$.

Remark 2.19. In addition to the number of blocks and the positions of rlm's, the above bijection also preserves (1) the size of each block, (2) the smallest element in each block, and (3) the elements in the last block. 
Below is an example of the bijection when $n=15$ and $k=6$ :

$$
\begin{aligned}
& \pi=\pi_{0}=123443552166543 \rightarrow \pi_{1}=123443551266543 \rightarrow \pi_{2}=123441552366543 \rightarrow \\
& \pi_{3}=123412553466543 \rightarrow \pi_{4}=123412534566543=f(\pi) .
\end{aligned}
$$

\section{Conclusion}

The general problem of enumerating the partitions of $[n]$ avoiding two (or more) patterns of length four or more seems to be difficult, with explicit solutions not possible in many cases. One could nonetheless attempt to describe all of the distinct Wilf equivalence classes for two patterns with given lengths (which would extend the work done in [4] describing all of the equivalence classes for a single pattern up to length seven). Here, we have identified eight subsets of the partitions of $[n]$ whose members avoid two classical patterns, each of which is counted by $C_{n}$ and has a refinement counted by $N_{n, k}$. We also have given explicit bijections from the classes (1), (3), (6), and (7) in Theorem 1.1 above to $(2),(4),(7)$, and (8), respectively. We leave the task of finding bijections between the remaining classes as a challenge to the reader. It would also be interesting if direct bijections with Dyck paths (or other structures enumerated by $C_{n}$ ) could be found in some of the more difficult cases. Furthermore, based on numerical evidence, there are no other pattern pairs $u$ and $v$ of lengths four and five such that $p_{n}(u, v)=C_{n}$ for all $n$, upon sorting through all of the possible cases for $u$ and $v$. Thus, there are no other members of the Wilf equivalence class for partitions corresponding to $C_{n}$ involving a pattern of length four and another of length five. The bijections, though, used to prove Propositions 2.2, 2.9 , and 2.18 can be extended to show the equivalence of some infinite families of pattern pairs, the details of which we leave to the interested reader. However, we were unable to enumerate, in general, the number of partitions of $[n]$ avoiding these pattern pairs.

Proposition 3.1. If $n, k \geq 0$, then

$$
\begin{aligned}
& \text { (i) } p_{n, k}\left(12^{i}, 123 \cdots j 23 \cdots j\right)=p_{n, k}\left(12^{i}, 123 \cdots j j(j-1) \cdots 2\right), \quad i, j \geq 2, \\
& \text { (ii) } p_{n, k}\left(121^{i}, 12 \cdots j 21\right)=p_{n, k}\left(121^{i}, 12 \cdots j 12\right), \quad i \geq 2, j \geq 3 \\
& \text { (iii) } p_{n, k}\left(1^{i} 2,12123\right)=p_{n, k}\left(1^{i} 2,12213\right), \quad i \geq 2 .
\end{aligned}
$$

\section{References}

[1] C. Banderier, M. Bousquet-Mélou, A. Denise, P. Flajolet, D. Gardy, and D. GouyouBeauchamps, Generating functions for generating trees, (Formal Power Series and Algebraic Combinatorics, Barcelona, 1999), Discrete Math. 246:1-3 (2002) 29-55.

[2] M. Bóna, The permutation classes equinumerous to the smooth class, Electron. J. Combin. 5 (1998) \#R31.

[3] A. Goyt, Avoidance of partitions of a three element set, Adv. in Appl. Math. 41 (2008) 95-114. 
[4] V. Jelínek and T. Mansour, On pattern-avoiding partitions, Electron. J. Combin. 15 (2008) \#R39.

[5] M. Klazar, On abab-free and abba-free set partitions, European J. Combin. 17 (1996) $53-68$.

[6] D. E. Knuth, The Art of Computer Programming, Vol's. 1 and 3, Addison-Wesley, Reading, Mass. 1968, 1973.

[7] P. A. MacMahon, Combinatorial Analysis, Vol's. 1 and 2, Cambridge University Press 1915, 1916; reprinted by Chelsea, 1960.

[8] T. Mansour and A. Vainshtein, Counting occurrences of 132 in a permutation, Adv. in Appl. Math. 28:2 (2002) 185-195.

[9] T. V. Narayana, Sur les treillis formés par les partitions d'une unties et leurs applications à la théorie des probabilités, C. R. Acad. Sci. Paris 240 (1955) 1188-1189.

[10] J. Noonan and D. Zeilberger, Counting permutations with a prescribed number of forbidden patterns, Adv. in Appl. Math. 17 (1996) 381-407.

[11] A. Robertson, H. Wilf, and D. Zeilberger, Permutation patterns and continued fractions, Electron. J. Combin. 6 (1999) \#R38.

[12] B. E. Sagan, Pattern avoidance in set partitions, Ars Combin. 94 (2010) 79-96.

[13] R. Simion and F. W. Schmidt, Restricted permutations, European J. Combin. 6 (1985) 383-406.

[14] N. J. Sloane, The On-Line Encyclopedia of Integer Sequences, http://oeis.org.

[15] R. P. Stanley, http://www-math.mit.edu/rstan/, "Enumerative CombinatoricsCatalan addendum".

[16] D. Stanton and D. White, Constructive Combinatorics, Springer, New York 1986.

[17] R. A. Sulanke, The Narayana distribution, J. Statist. Plann. Inference 101 (2002) 311-326.

[18] C. Wagner, Generalized Stirling and Lah numbers, Discrete Math. 160 (1996) 199218.

[19] J. West, Generating trees and the Catalan and Schröder numbers, Discrete Math. 146 (1995) 247-262.

[20] H. S. Wilf, generatingfunctionology, Academic Press, Inc., 1994.

[21] D. Zeilberger, Enumeration schemes, and more importantly, their automatic generation, Ann. Comb. 2 (1998) 185-195. 